\title{
La régénération osseuse guidée : résultats, limites et perspectives
}

\section{Guided bone regeneration: results,} limitations and prospects

\section{MOTS-CLES : \\ - Régénération osseuse guidée, implant, gain osseux, membrane, os autogène, substituts osseux, complications. \\ KEYWORDS: \\ - Guided bone regeneration, implant, bone augmentation, membrane, autogenous bone, bone substitutes, complications.}

\section{Résumé}

Le grand défi en implantologie est d'obtenir des résultats stables et satisfaisants tant sur le plan esthétique que fonctionnel. Afin d'atteindre ce but, la création d'un volume osseux suffisant avant ou durant la pose des implants est devenue une règle incontournable chaque fois que le chirurgien dentiste est face à un défaut osseux péri-implantaire. La technique de régénération osseuse guidée (ROG) est la plus documentée dans la littérature et ses résultats en termes d'augmentation du volume osseux tel qu'ils seront analysés dans notre article montrent une fiabilité et un succès à long terme. Toutefois, comme pour toute technique la ROG a certaines limites et peut être malmenée par certains échecs. Les différents types de membrane et de substituts osseux utilisés dans cette technique seront présentés dans cet article et illustrés par trois cas cliniques.

\section{Abstract}

The challenge in oral implantology is to obtain adequate and long-term results either on the aesthetic or functional point of view. In order to achieve this goal, bone gain before or at implant placement, is needed each time we are faced to a bony defect. Guided bone regeneration (GBR) is the most documented between bone augmentation techniques in the literature and predictability of longterm results will be analyzed in this paper. Nevertheless, as for any other techniques, GBR has some limitations and failures. Different membranes and bone substitutes used in this approach will be presented and illustrated by three clinical cases.
AOS 2013;261:11-21 DOI: $10.1051 / \mathrm{aos} / 2013103$ (C) EDP Sciences 2013
Hadi ANTOUN, Exercice exclusif en implantologie et parodontologie, 11 bis, avenue Mac-Mahon, 75017 Paris.

Michel KAROUNI, CES Biologie de la bouche, Paris V, CES Parodontologie, Paris VII.

Bouchra SOJOD, CES Biologie de la bouche, Paris V, CES Parodontologie, Paris VII, université Saint-Joseph de Beprouth.

\section{INTRODUCTION}

La cicatrisation osseuse post-extractionnelle s'accompagne d'une perte osseuse aussi bien verticale que vestibulo-linguale (VL). Différentes études ont montré à un an $50 \%$ de résorption dans le sens VL (Schroeder,
1986 [45], Schropp et al., 2003 [46]) et une perte plus accentuée en vestibulaire (Pietrokovski, 1967 [40], Araujo et Lindhe, 2005[4], Pietrokovski, 2007 [41]). De plus, différentes pathologies dentaires et/ou parodontales vont engendrer une résorption osseuse plus ou moins importante. Parmi celles-ci, on peut citer 
les lésions endodontiques, parodontales, traumatiques ou encore tumorales. Face à ce constat, la réhabilitation d'un édentement par une restauration implanto-portée va nécessiter un apport osseux et muqueux afin de retrouver au plus près la situation initiale.

Alors qu'il y a quelques années la pose d'implants était quasi impossible en absence de volume osseux suffisant, à l'heure actuelle, le recours à la solution implantaire dans de telles conditions est devenu accessible grâce à l'avènement des techniques d'augmentation osseuse tant horizontale que verticale du lit périimplantaire. Parmi ces techniques, nous citerons : les greffes d'apposition, les greffes d'interposition, l'expansion, la distraction, les greffes de sinus ou encore la régénération osseuse guidée (ROG).

La ROG que nous allons exposer dans cet article a été introduite par Dahlin et al. en 1988 [13] et elle est fondée sur le principe d'exclusion cellulaire par l'utilisation de membrane. Par la suite, Buser et al. (1990) [7] ont proposé des techniques modifiées afin d'optimiser cette régénération. Ces travaux ont permis à partir des années 1990 un élargissement net du champ d'application de l'implantologie.

\section{PRINCIPES BIOLOGIQUES DE LA RÉGÉNÉRATION OSSEUSE GUIDÉE}

La théorie de compartiments des tissus a abouti à la technique de la régénération tissulaire guidée (RTG) car les cellules colonisent les surfaces selon le principe "premières arrivées, premières servies ». Cette théorie a mis en œuvre des moyens de contrôle de la migration cellulaire par des filtres et plus tard par des membranes. Le concept de la ROG est fondé sur les bases de la RTG (Nyman et al., 1980 [36], Karring et al., 1980 [29], 1984, Melcher et al., 1976 [33]) et sous sa forme classique, la ROG fait appel à des barrières physiques sous forme de membrane. Ces barrières empêchent la colonisation du défaut osseux par les tissus mous conjonctifs et épithéliaux et permettent ainsi seulement aux cellules à pouvoir ostéogénique d'envahir l'espace cicatriciel (Dahlin et al., 1988 [13], Hammerle et al., 1995 [22]).

D’après Buser et al. (2009) [9], les membranes ont un triple rôle :

$\checkmark$ prévenir la prolifération des cellules à partir de la muqueuse de recouvrement et favoriser la migration des cellules issues des espaces médullaires dans le caillot habitant le site ;

I stabiliser la greffe osseuse et le caillot ;

$\checkmark$ sopposer à la résorption de la crête.

Dans cette optique, plusieurs types de membranes résorbables et non résorbables ont été utilisés dans des études expérimentales et cliniques. Les caractéristiques d'une membrane sont surtout : la biocompatibilité, l'exclusion cellulaire, l'intégration tissulaire, le maintien et la création de l'espace cicatriciel, la facilité de manipulation et la susceptibilité aux complications (Karring et al., 1993 [28]).

\section{MEMBRANES NON RÉSORBABLES}

Les caractéristiques des membranes non résorbables sont surtout l'inertie biologique, la flexibilité, la stabilité chimique et la micro-porosité asymétrique. En revanche, ce type de membrane présente quelques inconvénients tels que : la nécessité de fixation par des vis et d'une deuxième intervention pour la déposer, ainsi que le risque d'exposition de membrane et l'infection qui peut en découler.

Depuis les travaux de l'équipe scandinave (Nyman et al., 1989 [38]), l'utilisation des membranes non résorbables type polytétrafluoroéthylène expansé (ePTFE) a connu une expansion croissante et, selon un grand nombre d'études, son utilisation pour la ROG aboutit à des résultats très prédictibles (Hammerle et Jung, 2003 [20], Dahlin, 1995 [14], Nyman et al., 1990 [37]). Jovanovic et al., 1995 [26], ont comparé les membranes ePTFE renforcées titane (TR) aux membranes ePTFE standard, un gain osseux moyen de $3,3 \mathrm{~mm}$ et de 2,4 $\mathrm{mm}$ respectivement ont été obtenus (cas clinique $\mathbf{n}^{\circ} \mathbf{1}$, fig. 1 à 16). Cette étude montre que les membranes ePTFE (TR) entraînent une augmentation de la largeur de la crête et une stabilisation du caillot sanguin sans utilisation de greffe osseuse dans le cas de déhiscence péri-implantaire.

D’après Becker et al. (1999) [5], l'utilisation de membranes ePTFE dans le comblement de déhiscences et fenestrations péri-implantaires montre un taux de survie cumulatif de $77 \%$ au maxillaire et $84 \%$ à la mandibule, ces résultats sont moins prédictibles que l'EII associée à une ROG. Dans ce dernier cas, les taux de survie cumulatifs sont de 93,8\% au maxillaire et à la mandibule. Cependant, le problème majeur des membranes non résorbables réside dans le risque d'exposition et la résorption osseuse qui peut accompagner une telle complication.

Nowzari et al. (1995) [35] ont comparé les résultats de la ROG en présence et en absence d'exposition de membrane ePTFE. Ils ont démontré que l'exposition de la membrane entraîne une persistance du défaut osseux péri-implantaire de 3,2 $\mathrm{mm}$ en moyenne et des signes d'inflammation et de suppuration associés à un pourcentage élevé de bactéries pathogènes (Aa, $\mathrm{Pg}, \mathrm{Pi}, \mathrm{Bf})$. De même, Machtei et al. en 2001 [32] montrent que l'exposition de la membrane ePTFE diminue de 6 fois le gain osseux.

\section{GREFFONS OSSEUX}

Lutilisation de membrane seule en ROG en per-implantaire est indiquée dans le cas de petits défauts osseux ne dépassant pas $3 \mathrm{~mm}$ (Jovanovic et al., 1995 [26]). Pour la reconstitution de gros défauts préalablement à la pose d'implant, selon la plupart des études, l'utilisation d'une membrane seule ne suffit pas à cause du risque d'effondrement de celle-ci et par la suite 


\section{Cas clinique $n^{\circ} 1$}

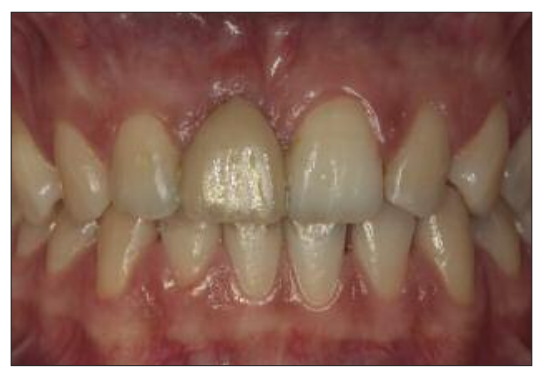

$\triangleleft$ Fig. 1 :

Mme P.B. est adressée pour la pose d'un implant au niveau de la 11 Son bridge collé, se descellant régulièrement, ne lui apportait plus satisfaction.
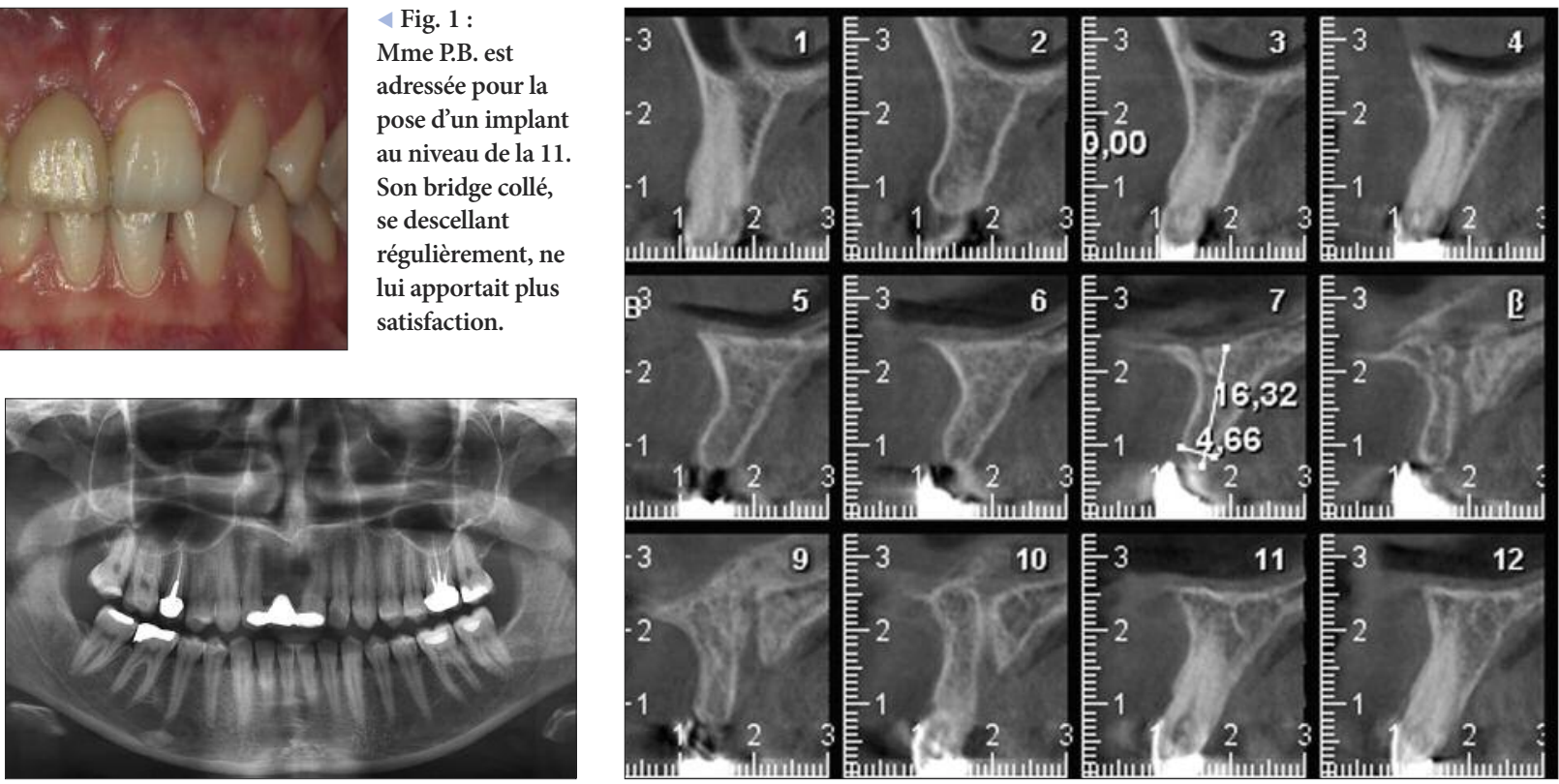

$\triangle$ Fig. 2 et $3 \Delta$ :

Examen radiologique montrant une perte osseuse horizontale permettant de poser un implant. Cependant, la concavité, présente, nécessite une régénération osseuse guidée horizontale associée à la pose d'un implant en 11.

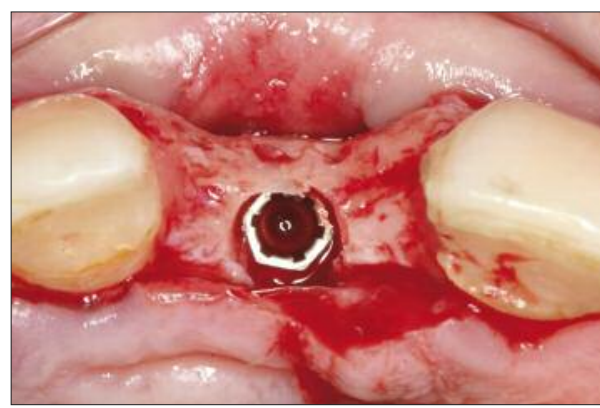

$\triangleleft$ Fig. 4 :

Pose d'un implant NobelSpeedy ${ }^{\circ} 4$ x 13 $\mathrm{mm}$ (Nobel Biocare) en 2009.

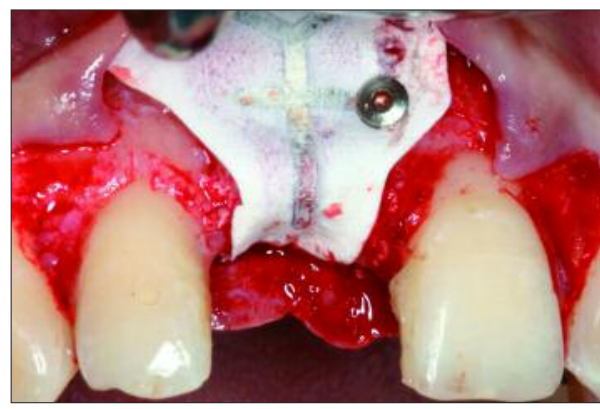

4 Fig. 6 :

Comblement du défaut osseux avec un matériau d'origine bovine (Bio-Oss', Geistlich) et pose d'une membrane Gore-Tex renforcée titane fixée par des clous titane.

$\nabla$ Fig. 8 à 10 v:

Réentrée à 6 mois ; bonne intégration de la membrane et après sa dépose on peut noter l'augmentation osseuse horizontale permettant de retrouver le volume osseux perdu.
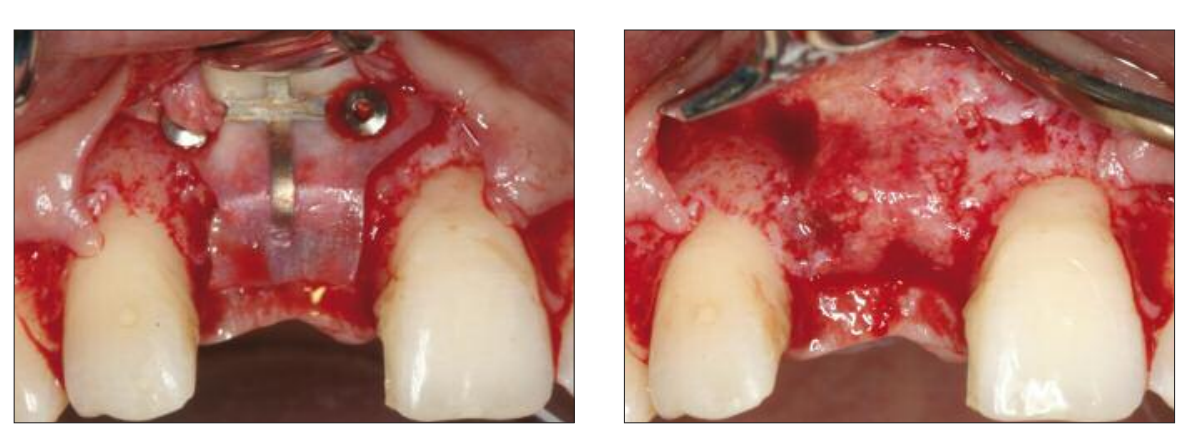

$\triangleleft$ Fig. 5 :

Vue vestibulaire montrant le défaut osseux ainsi que les perforations corticales réalisées favorisant l’angiogenèse.

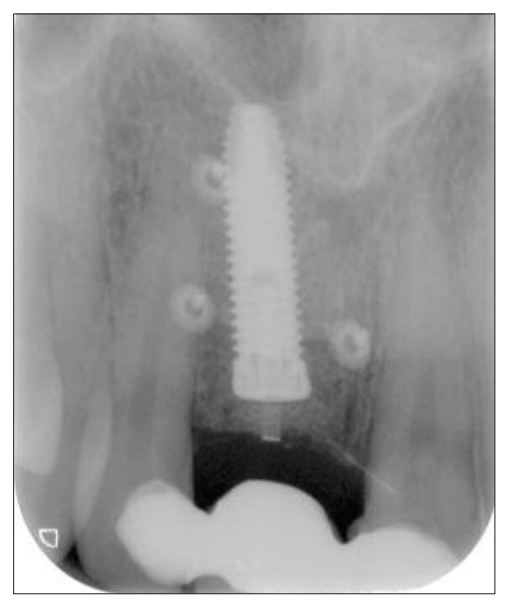

$\triangleleft$ Fig. 7 :

Radiographie de contrôle à 2 mois montrant la position de l'implant et le tissu minéralisé associé à la membrane recouvrant l'implant.

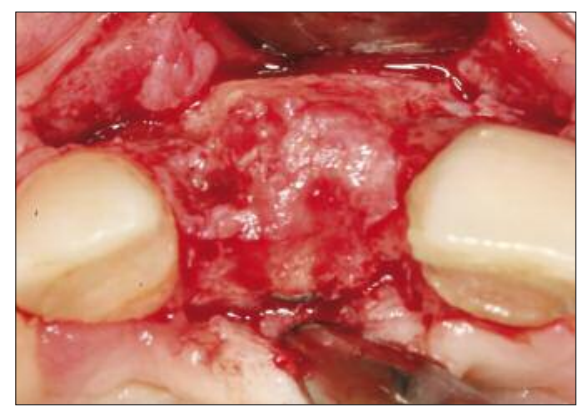




\section{Cas clinique $n^{\circ} 1$}

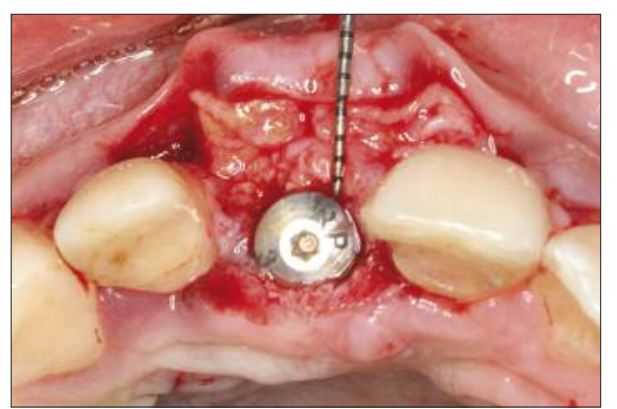

$\triangleleft$ Fig. 11 :

Connexion du pilier de cicatrisation et association d'une greffe conjonctive enfouie en vestibulaire après un prélèvement au palais.

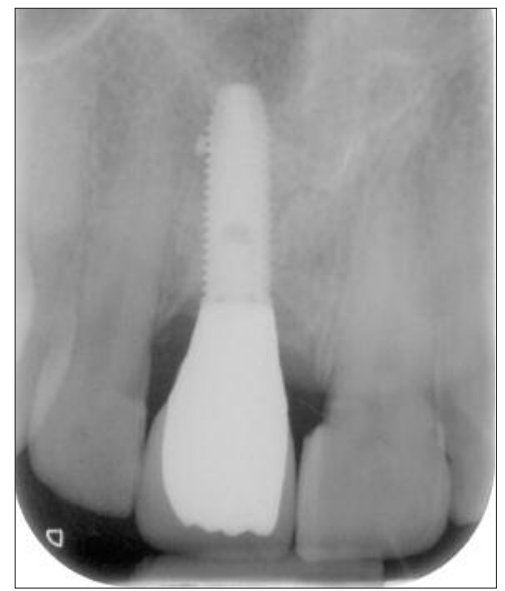

4 Fig. 13 :

Radiographie de contrôle avec la couronne d'usage à plus de 3 ans. À noter la stabilité du niveau osseux proximal.

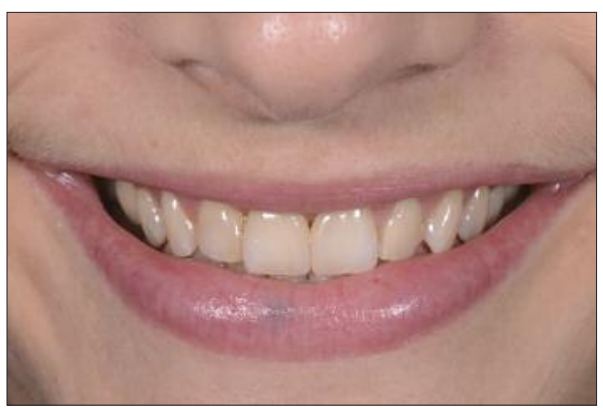

$\measuredangle$ Fig. 15 et $16 \triangleright$ : Sourire harmonieux de la patiente satisfaite du résultat fonctionnel et esthétique obtenu. (Prothèse du Dr Pierre Cherfane). de la disparition de l'espace cicatriciel susceptible de créer l'environnement adéquat pour la néoformation osseuse (Sandberg, 1993 [44], Kostopoulos, 1994 [30]). À l'exception des membranes rigides, l'interposition d'un greffon osseux entre la membrane et le défaut osseux s'avère nécessaire et ceci pour les raisons suivantes (Buser et al., 2009 [9]) :

\supporter la membrane pour éviter l'effondrement ; I jouer le rôle d'échafaudage pour la croissance osseuse dans le site receveur ;

I stimuler la croissance osseuse ;

I assurer une protection mécanique contre la pression du recouvrement des tissus mous ;

$\checkmark$ protéger le volume augmenté de la résorption.

Ces greffons osseux peuvent avoir plusieurs origines : autogène, humaine, animale ou synthétique.

Pendant plusieurs années, l'os autogène a été considéré comme le gold standard grâce à son potentiel ostéogénique (Chiapasco et al., 1999 [10], Buser et al., 1998 [8], Antoun et al., 2000 [1], Pallesen et al., 2002 [39], Springer et al., 2004 [49]). Néanmoins des taux de résorption de $60 \%$ des blocs autogènes ont été démontrés dans certaines études ainsi que des risques de morbidité du site donneur (HjortingHansen E., 2002 [24], Antoun et al., 2007 [3]). Le recouvrement de ces blocs osseux par des membranes résorbables ou non résorbables limite fortement leur résorption (Antoun et al., 2001 [2]). L'utilisation de substituts osseux, de par l'absence de morbidité, trouve de plus en plus d'intérêt et des résultats comparables avec l'os autogène dans les régénérations osseuses guidées sont reportés (Fontana et al., 2008 [15], Yeh et al., 2003 [53], Simion et al., 1996 [48]).

\section{MEMBRANES RÉSORBABLES}

Les membranes résorbables utilisées pour la ROG sont de deux types collagéniques (Avitene ${ }^{\varpi}$, Paroguide ${ }^{\varpi}$, Biostite $^{\oplus}$, Bio-Gide ${ }^{\oplus}$, Zyderm ${ }^{\oplus}$, Perio-Barrier ${ }^{\circledast}$, Periogen ${ }^{\circledR}$ BioMend $^{\circledast}$ ) et synthétiques (Guidor ${ }^{\circledast}$ Atrisorb $^{\circledR}$ Vicryl $^{\circledR}$ Periodontal Mesh, Resolut ${ }^{\mathrm{Tm}} /$ Resolut XT).

La membrane résorbable doit avoir un temps de dégradation compatible avec le délai nécessaire pour 
la formation osseuse. La dégradation ne doit avoir lieu que quand l'ossification du défaut osseux est atteinte complètement et ceci pour maintenir l'espace pendant une période de 3 à 9 mois.

\section{Avantages :}

$\checkmark$ pas de chirurgie de retrait de la membrane;

I simplification de la procédure chirurgicale ;

$\checkmark$ diminution des complications post-opératoires.

\section{Inconvénients :}

pas de contrôle de la durée de fonction de la barrière ; I possibilité d'interférence entre la résorption/cicatrisation et la régénération osseuse ;

Inécessité d'un matériau supportant la membrane.

La littérature montre des résultats plus ou moins favorables avec l'utilisation des membranes résorbables (Sandberg et al., 1993 [44], Zellin et al., 1995 [54], Brunel et al., 1998 [6]). Les membranes Bio-Gide ${ }^{\circledast}$ (Geistlich) sont les plus documentées, et elles sont toujours associées à un greffon osseux (cas clinique $\mathbf{n}^{\circ} 2$, fig. 17 à 24).

Hammerle et al. (2001) [21] ont montré que la ROG faisant recours à une membrane Bio-Gide ${ }^{\circledast}$ et du BioOss ${ }^{\oplus}$ permet une diminution du défaut péri-implantaire de 2,5 mm à 7 mois. Des gains osseux de $4 \mathrm{~mm}$ en moyenne ont été rapportés par le même auteur, en 2008 [22], suite à l'utilisation de la même technique. Afin doptimiser les propriétés de biodégradation des membranes en collagène, des membranes en collagène réticulé ont été mises sur le marché. Friedman et al. (2002) [16], par une étude clinique randomisée, ont montré que la barrière membrane Ossix combinée au BioOss s'accompagne d'une régénération osseuse histologiquement qualitative et comparable à celle obtenue par la membrane en ePTFE.

Dans une étude récente, Tal et al. (2008) [50] ont montré que les membranes collagènes réticulés (CLM) sont plus résistantes à la dégradation que les membranes non croisées (NCLM) et maintiennent une intégrité tissulaire tout au long de létude. Les deux types de membranes ne résistent pas à la dégradation dans le cas d'exposition avec pour les CLM un plus grand pourcentage d'exposition.

Les membranes synthétiques sont aussi bénéfiques que les autres membranes (Geurs et al., 2008 [19]) et montrent de meilleures propriétés mécaniques et une résorption plus lente que les membranes collagènes

\section{Cas clinique $\mathrm{n}^{\circ} 2$}

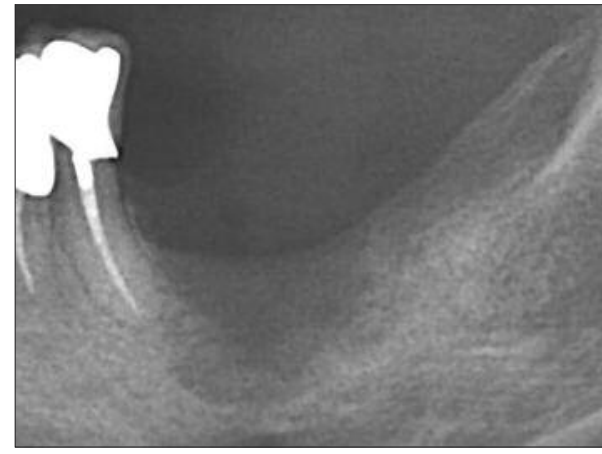

$\triangle$ Fig. 17 :

Après une perte d'implants au niveau du secteur 3, Mme L.A. souhaite à nouveau retrouver des dents fixes. La radiographie montre l'absence de 36 et 37 avec une perte de substance évidente.

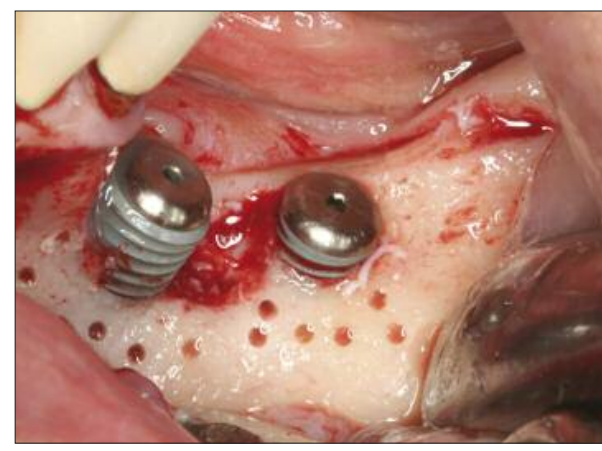

$\triangle$ Fig. 19 :

La mise en place des implants Branemark Mk3

$5 \times 8,5 \mathrm{~mm}$ en 37 et Nobel Speedy $6 \times 8,5 \mathrm{~mm}$ en 37 (Nobel Biocare) a entraîné des déhiscences osseuses importantes exposant une grande partie des spires en particulier au niveau de la 36 . Réalisation de perforations corticales avant la greffe osseuse.

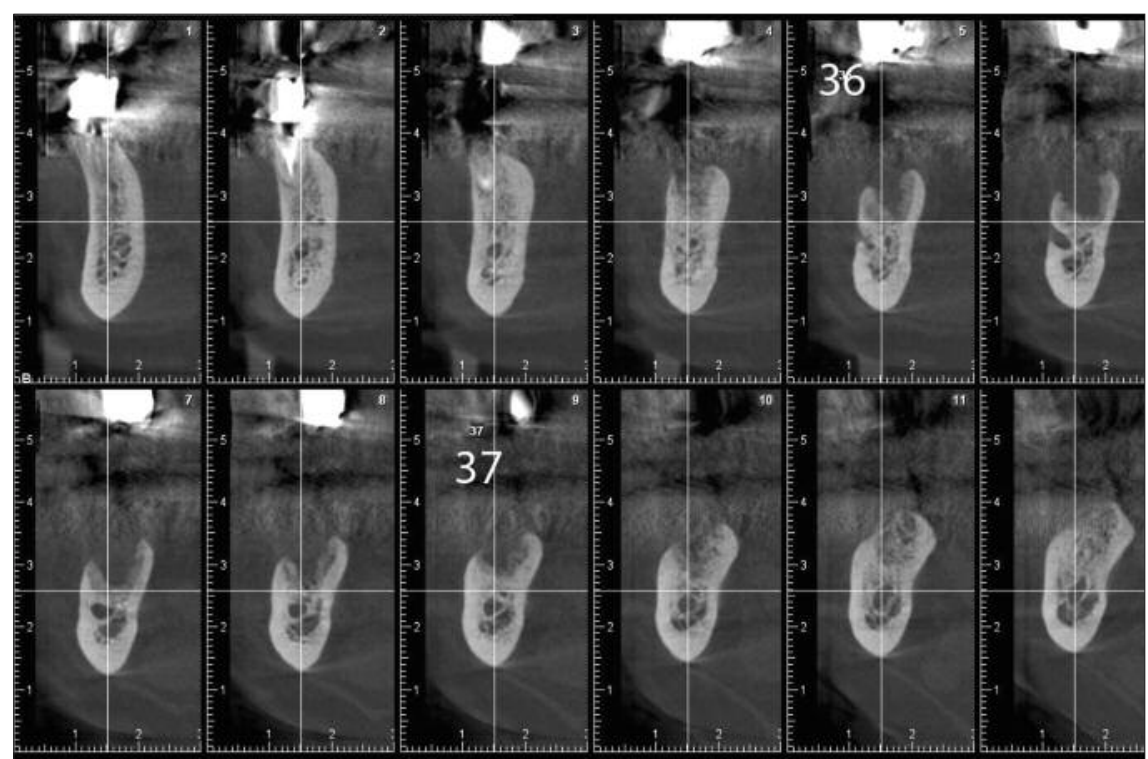

$\triangle$ Fig. 18 :

L'examen scanner montre une résorption osseuse horizontale et verticale qui nécessite une augmentation osseuse. La configuration de la crête permet de poser l'indication d'une ROG perimplantaire.

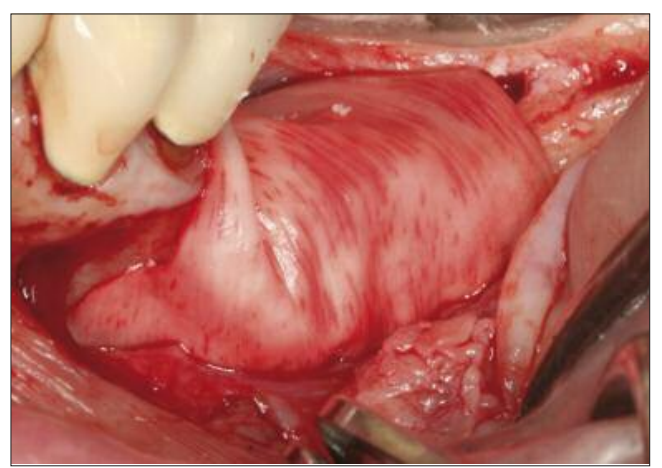

$<$ Fig. 20 :

Comblement du défaut avec de l'os autogène en premier prélevé au niveau de la ligne oblique externe, recouvert d'hydroxyapatite d'origine bovine et pose d'une membrane résorbable (Bio-Gide ${ }^{\circledR}$, Geistlich). 


\section{Cas clinique $n^{\circ} 2$}
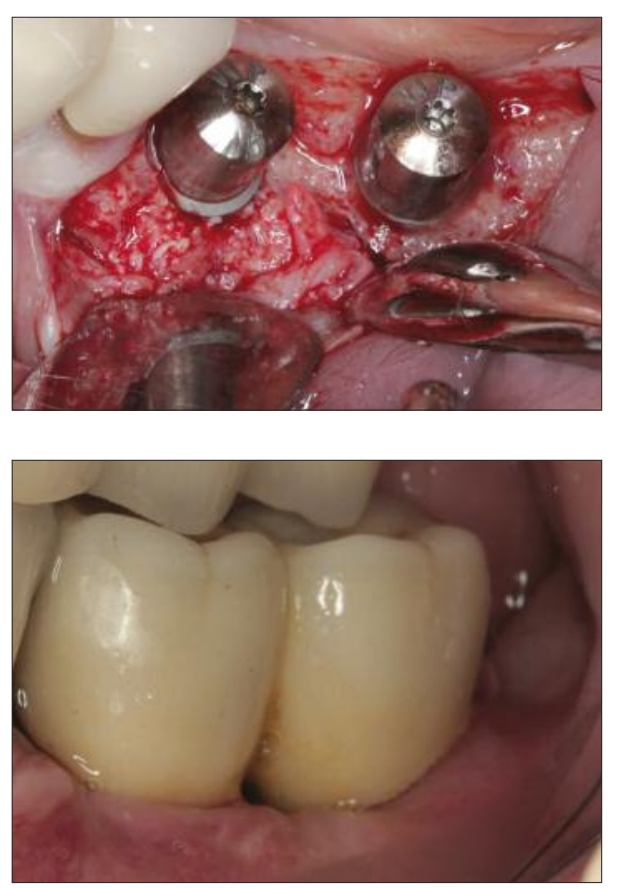

4 Fig. 22 et 23 :

La prothèse d'usage est transvissée directement sur les implants. Ceci permet une démontabilité facilitée pour la maintenance et en cas de complication.

(Prothèse du

Dr Pierre Cherfane).
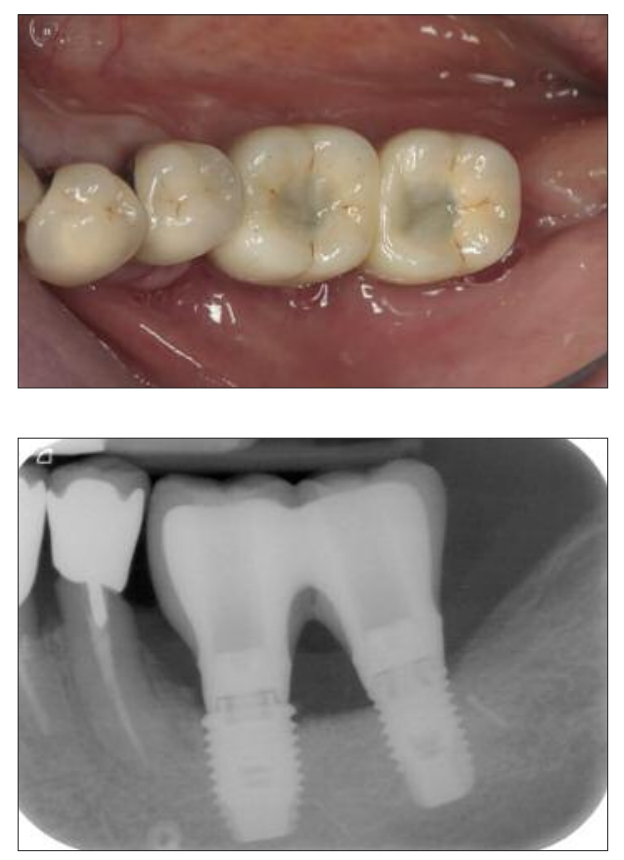

$\triangle$ Fig. 24 :

Contrôle radiographique à

2 ans montrant une stabilité du niveau osseux du côté proximal des implants.

(Gentile et al., 2011 [18]). En revanche, les membranes synthétiques comme la PLA (polylactic-acid) peuvent induire des réactions inflammatoires et parfois même des résorptions osseuses (Gentile et al., 2011 [18], Polimeni et al., 2008 [42]).

\section{MEMBRANES RÉSORBABLES VS MEMBRANES NON RÉSORBABLES}

La différence entre les membranes résorbables et non résorbables réside dans la réaction tissulaire en cas d'exposition de membrane et la nécessité de greffon osseux. Une étude par Moses et al. (2008) comparant différents types de membranes dans le traitement de déhiscence, montre que l'exposition prématurée des membranes résulte en une mauvaise cicatrisation osseuse. Quelques membranes barrières, comme les membranes Ossix semblent capables de continuer la guérison gingivale même lorsquelles sont prématurément exposées. Cependant, elle n'est plus commercialisée.

Selon Chiapasco et al. (2009) [12], le taux d'exposition et d'infection sont plus importants dans le cas de membranes non résorbables (20\%) que dans le cas de membranes résorbables (5\%), et selon les mêmes auteurs, dans presque tous les cas, l'utilisation de membranes résorbables nécessitait l'interposition d'un matériau de comblement osseux.

Lorenzoni et al. (2002) [31], par une étude de suivi ont évalué les paramètres cliniques et radiographiques d'implants dentaires placés en association avec la régénération osseuse guidée avec différents types de membranes barrières. Une moyenne de perte osseuse globale de 0-3,5 mm et une perte osseuse durant la phase de cicatrisation de $0,8 \mathrm{~mm}$ ont été notées. Une exposition prématurée de la membrane a résulté en une perte osseuse crestale de 3-4 mm.

\section{ARBRE DÉCISIONNEL ET INDICATIONS}

Le choix de la technique appropriée se fait en fonction de plusieurs facteurs. James et Myron (1995) ont essayé de regrouper les facteurs qui orientent le choix de la technique appropriée afin d'augmenter la prédictibilité de la ROG. Elle peut être utilisée pour la reconstruction de tous les types de défauts osseux extractionnels. Les défauts osseux qui ne permettent pas le maintien d'un espace nécessitent l'apport d'un biomatériau.

Des facteurs liés au patient, à l'anatomie du défaut, des facteurs per-, pré- et post-chirurgicaux sont considérés comme étant très pertinents dans la prise de décision (Tableau I).

Jensen et al. (2009) [27], par une revue systématique, ont essayé d'évaluer l'efficacité des différents protocoles de greffe dans le traitement de défauts osseux localisés. Selon cette équipe, il semblerait que les meilleurs protocoles soient les suivants :

Iéfaut de type déhiscence et fenestration : substitut osseux recouvert par une membrane, particules d'os autogènes avec ou sans membrane, et membrane non résorbable sans greffon osseux;

$\checkmark$ augmentation horizontale de crête : bloc d'os autogène seul ou associé à un substitut osseux avec ou sans l'utilisation de membrane ;

$\checkmark$ augmentation verticale : greffe d'os autogène, bloc ou particules supportées par une membrane.

Pour les défauts dans le sens horizontal, Fu JH et Wang HL (2011) [17], proposent un arbre décisionnel en fonction de la largeur initiale de la crête et de la stabilité primaire de l'implant (Tableau II). 


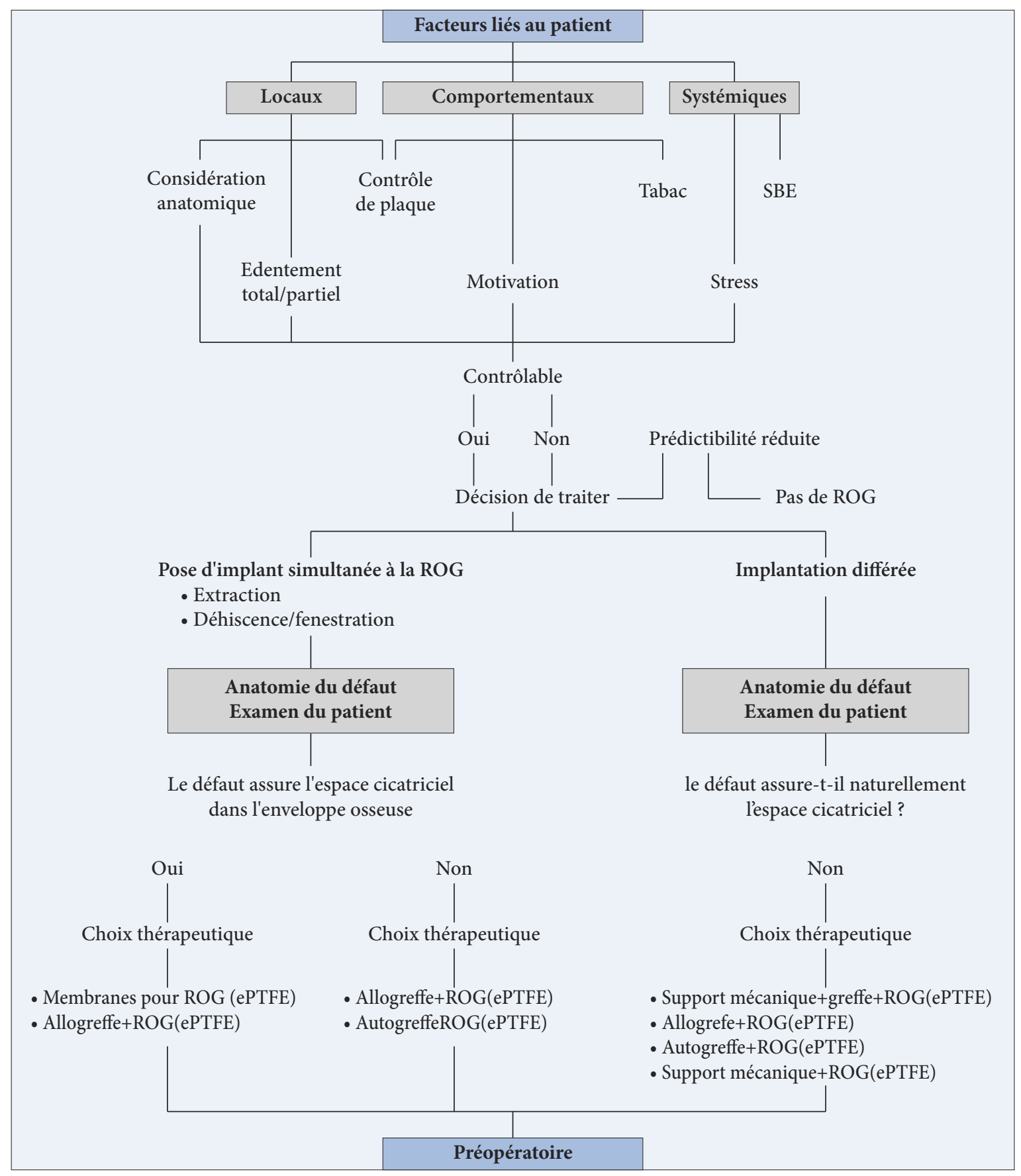

$\varangle$ Tableau I : Arbre décisionnel des facteurs influençant la thérapeutique de Mellonig J.T. et Nevins M. (1995) [34].

\begin{tabular}{|c|c|c|c|}
\hline \multicolumn{4}{|c|}{ Largeur vestibulo-linguale } \\
\hline \multicolumn{2}{|c|}{$\geq 3,5 \mathrm{~mm}$} & $<3,5 \mathrm{~mm}$ & $<3,5 \mathrm{~mm}$ \\
\hline \multirow{4}{*}{$\begin{array}{l}\text { Bonne } \\
\text { stabilité } \\
\text { primaire } \\
\text { Implantation } \\
\text { simultanée }\end{array}$} & $\begin{array}{c}\text { Pas de } \\
\text { stabilité } \\
\text { primaire }\end{array}$ & $\begin{array}{c}\text { Pas de stabilité } \\
\text { primaire }\end{array}$ & \multirow[t]{4}{*}{$\begin{array}{c}\text { Expansion } \\
\text { crestale }\end{array}$} \\
\hline & & Greffe d'apposition & \\
\hline & $\begin{array}{c}\text { Réalisation } \\
\text { en } 2 \text { temps } \\
\text { (implantation } \\
\text { différée) }\end{array}$ & $\begin{array}{c}\text { Épaisseur des tissus } \\
\geq 1,5 \mathrm{~mm}\end{array}$ & \\
\hline & & $\begin{array}{c}\text { Mandibule: Maxillaire : } \\
\text { autogène }\end{array}$ & \\
\hline
\end{tabular}

$\triangle$ Tableau II :

Arbre décisionnel des défauts dans le sens horizontal de Fu JH et Wang HL (2011) [17].
En ce qui concerne les défauts verticaux, l'examen de la littérature montre que les techniques le plus souvent proposées pour palier à ce problème sont : les greffes d'apposition, la distraction alvéolaire, la régénération osseuse guidée (cas clinique $\mathbf{n}^{\circ} 3$ ). D’après une revue systématique de Rochietta et al. en 2008 [43], la supériorité d'une technique précise par rapport à une autre n'est pas identifiée et ceci à cause du nombre limité de patients et de l'hétérogénéité des résultats des différentes études.

La distraction osseuse montre un meilleur pronostic en termes de maintenance du gain osseux et de résorption péri-implantaire par rapport à l'utilisation de membrane ePTFE associée à de los autogène (Chiappasco et al., 2004 [11]). Cependant, cette technique est très opérateur dépendant et nécessite une pratique régulière de cette approche et, en cas déchec, le séquestre osseux engendré aboutit à un défaut encore plus important. 
Pour ce qui est de la régénération du volume osseux péri-implantaire, Zitzman et al. (2001) [55] trouvent que l'utilisation de la ROG est indiquée quand le défaut initial péri-implantaire est supérieur à $2 \mathrm{~mm}$ dans le sens vertical. Chiapasco et al. (2009) [12] quant à eux trouvent dans une revue systématique que la ROG associée à la pose d'implant joue un rôle important dans les sites esthétiques. Il y aurait 3 complications esthétiques en cas d’absence de ROG péri-implantaire : 1. récession en vestibulaire, exposant quelques spires de l'implant ;

2. liseré grisâtre au niveau du collet gingival ;

13. absence de convexité de la crête alvéolaire en vestibulaire.

Tonetti et al. (2008) [51] montrent un gain osseux vertical suite à la ROG de $2-8 \mathrm{~mm}$, et une résorption osseuse péri-implantaire de 1,8-2 $\mathrm{mm}$ à 7 ans.
Dans une étude récente, Schwarz et al. (2012) [47] ont évalué l'effet du défaut osseux résiduel après ROG dans le cas de déhiscences sur la stabilité de la santé péri-implantaire après un suivi de 5 ans.

Un défaut osseux résiduel supérieur ou égal à $1 \mathrm{~mm}$ présente un plus grand risque de développer une péri-implantite et une récession gingivale peut y être associée compromettant ainsi le résultat esthétique.

\section{DISCUSSION ET CONCLUSION}

La régénération osseuse guidée est, sans conteste, une des plus grandes avancées en implantologie. C'est, sans doute, l'une des approches les mieux documentées dans cette discipline au niveau de la littérature mais

\section{Cas clinique $n^{\circ} 3$}
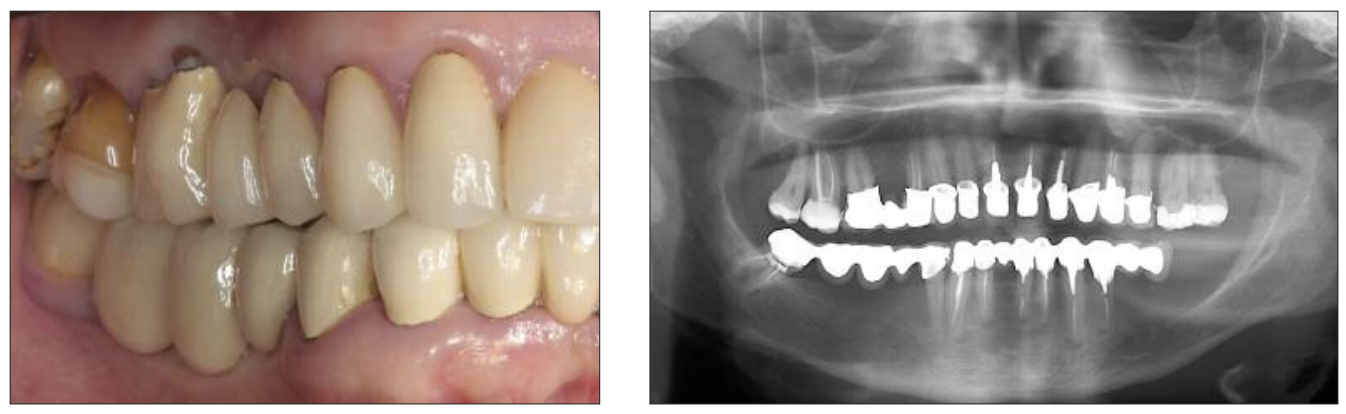

4 Fig. 25 et 26 :

Mme I.R. est adressée par son praticien traitant pour une pose d'implants au niveau des secteurs 3 et 4 . Le panoramique montre l'absence de 45,46 et 47 ainsi que 35 à 37 et l'indication de l'extraction de la 48 .
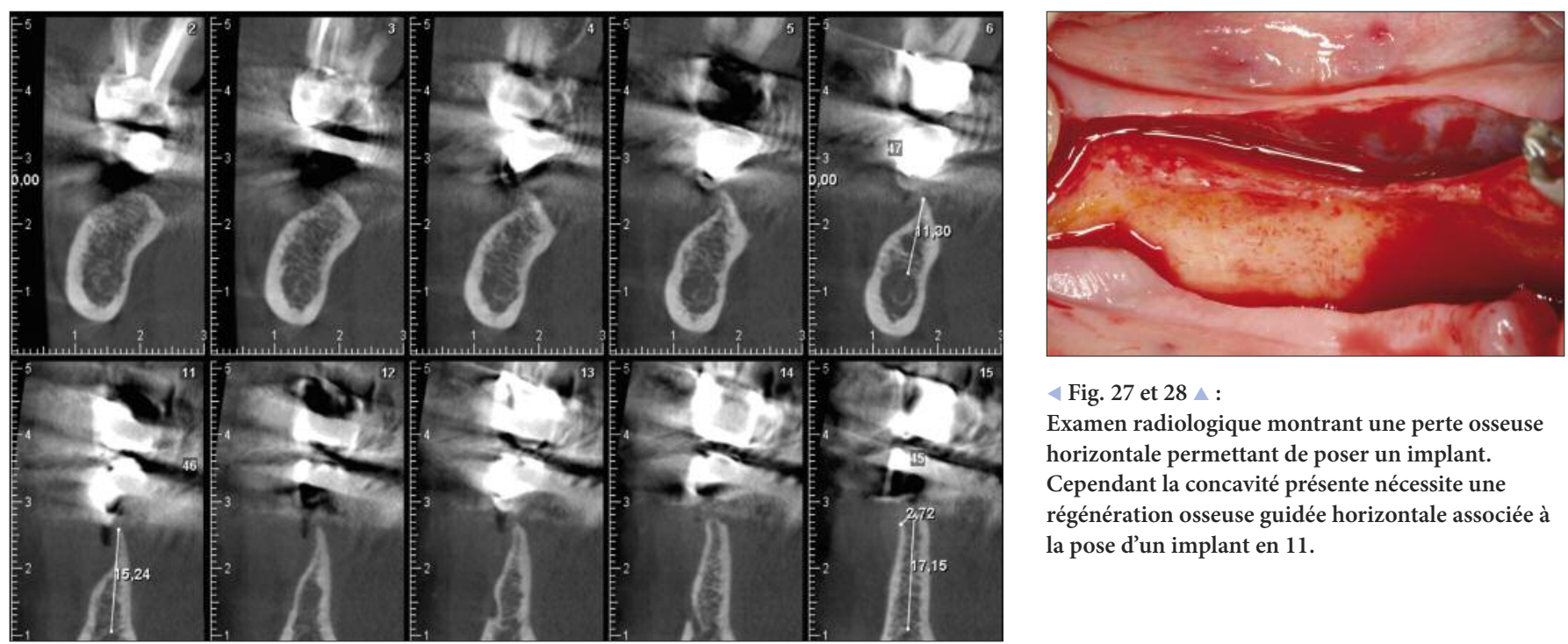

\section{$<$ Fig. 27 et $28 \triangle$ :}

Examen radiologique montrant une perte osseuse horizontale permettant de poser un implant. Cependant la concavité présente nécessite une régénération osseuse guidée horizontale associée à la pose d'un implant en 11.

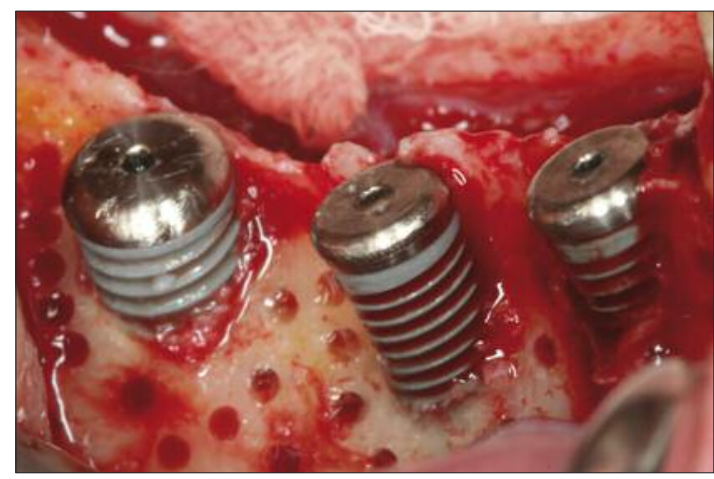

4 Fig. 29 :

La mise en place des implants

NobelSpeedy ${ }^{\oplus} 4$ x $11,5 \mathrm{~mm}$ sur $45 / 46$

et WP $5 \times 8,5 \mathrm{~mm}$ (Nobel Biocare ${ }^{\circ}$ )

dans une position optimale a

entraîné des déhiscences osseuses

importantes exposant une grande partie des spires. 

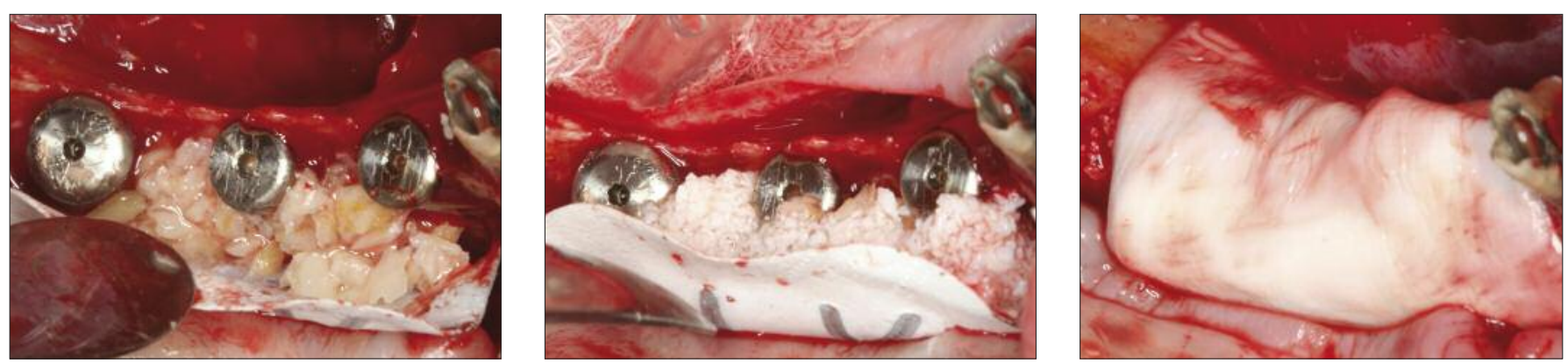

$\triangle$ Fig. 30 à $32 \Delta$ :

Comblement du défaut avec de l'os autogène broyé recouvert d'hydroxyapatite d'origine bovine et pose d'une membrane Gore-Tex renforcée titane, l'ensemble protégé d'une membrane résorbable (Bio-Gide ${ }^{\circledast}$, Geistlich).
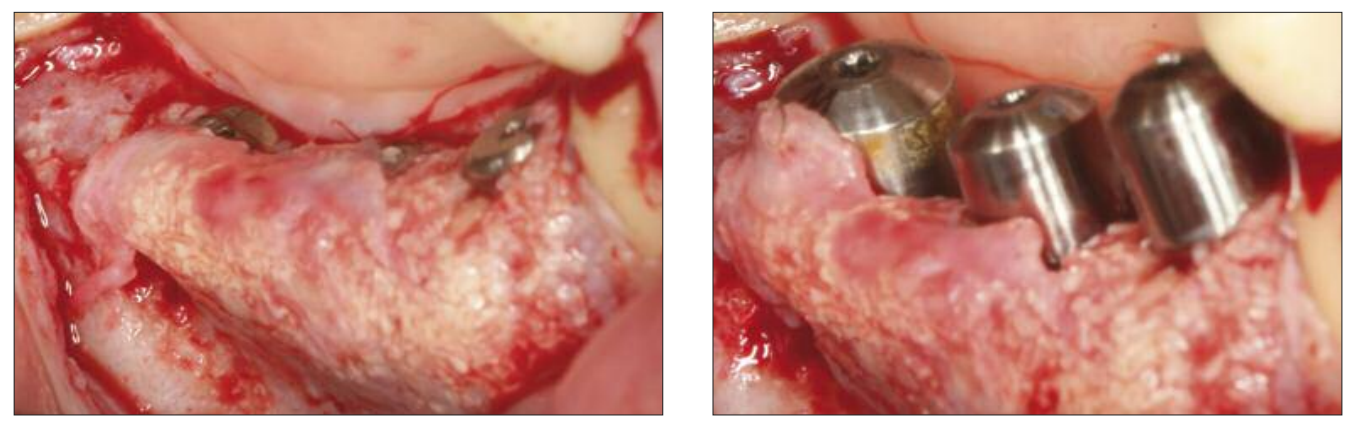

4 Fig. 33 et 34 :

Réentrée à 6 mois et pose des piliers de cicatrisation. Noter le recouvrement osseux obtenu comparativement à la fig. 29 montrant l'ensemble des spires exposées.

Fig. 35 et 36 :

Mise en place des couronnes d'usage et contrôle radiographique du niveau osseux proximal stabilisé après un remodelage sur 1 à 2 spires.

(Prothèse du

Dr Jean-Pierre Himpens).
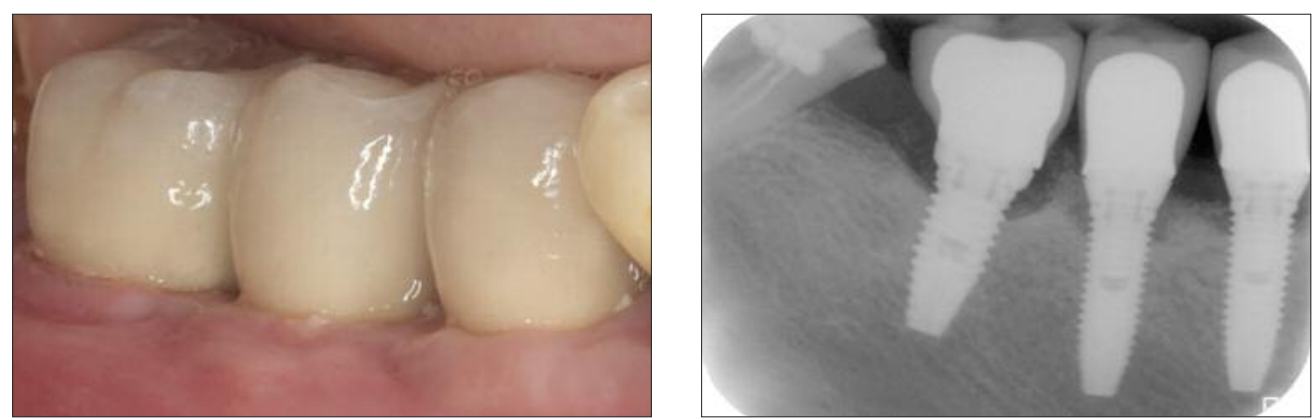

souvent malmenée par certains échecs, comme l'exposition des membranes non résorbables, aboutissant parfois à des situations critiques. Les résultats cliniques montrent que la ROG est une approche thérapeutique fiable dans le traitement des défauts péri-implantaires. Des augmentations osseuses verticales et horizontales peuvent être réalisées par la ROG, avec des degrés variables de succès.

En présence d'un défaut osseux compromettant soit le pronostic de l'implant soit le résultat esthétique, la ROG est notre premier choix thérapeutique quand il est possible d'aboutir à une bonne stabilité primaire ainsi quà une position et un axe implantaire en adéquation avec le projet prothétique.

Le choix d'une membrane résorbable associée à un substitut osseux est la première option choisie quand le défaut le permet. Quand il s'agit d'un défaut en dehors de son enveloppe osseuse, l'association d'os autogène semble incontournable. Si l'on veut éviter tout risque de morbidité et réduire les suites opératoires pour le patient, il faudrait associer une membrane non résorbable qui permet aussi d'assurer un échafaudage suffisant pour les augmentations osseuses en hauteur. Des études supplémentaires permettront d'élucider l'effet des facteurs liés au patient et au site dans l'augmentation de la prédictibilité des techniques de ROG. De plus, les propriétés de l'os régénéré à long terme restent à évaluer ainsi que les processus moléculaires mis en œuvre lors de la cicatrisation du site osseux régénéré dans l'intention de développer le concept d'ingénierie tissulaire basé sur de nouvelles technologiesd'assemblage cellulaires, moléculaires et matricielles. Ce concept nous permet dans l'avenir de pousser les limites des stratégies conventionnelles de régénération osseuse (Huang et al., 2004 [25], Umeda et al., 2009 [52]).

Lanalyse précise des cas est la clé de réussite de toute stratégie thérapeutique, tout en gardant à l'esprit que lobjectif commun du praticien et du patient est de mettre en œuvre une restauration implanto-prothétique qui répond le mieux aux exigences esthétiques et fonctionnelles. 


\section{Bibliographie}

[1] Antoun H, Abillama F, Daher F. Site de prélèvements osseux et reconstructions alvéolaires pré-implantaires. Implant 2000;66(4):257-368.

[2] Antoun H, Sitbon JM, Martinez H, Missika P. A prospective randomized study comparing two techniques of bone augmentation: onlay graft alone or associated with a menbrane. Clin Oral Implants Res 2001;12:632-9.

[3] Antoun H, Cherfane P, Billereau P. Morbidité du prélèvement osseux symphysaire. Implant 2007;13(4):249-60

[4] Araujo MG, Lindhe J. Dimensional ridge alterations following tooth extraction. An experimental study in the dog. J Clin Periodontol 2005;32:212-8.

[5] Becker W, Dahlin C, Lekholm, Bergstrom C. Five-year evaluation of implants placed at extraction and with dehiscences and fenestration defects augmented with ePTFE membranes: results from a prospective multicenter study. Clin Imp Dent Relat Res 1999;1(1);27-32.

[6] Brunel G, Benque E, Elharar F. Sansac C, Duffort JF, Barthet P, Baysse E, Miller N. Guided bone regeneration for immediate non-submerged implant placement using bioabsorbable materials in Beagle dogs. Clinical Oral Implants Res 1998; 9:303-12.

[7] Buser D, Bragger U, Lang NP, Nyman S. Regeneration and enlargement of jaw bone using guided tissue regeneration. Clin Oral Implants Res 1990;1:22-32.

[8] Buser D, A comparative study of osseointegration of titanium implants in autogenous and freeze-dried bone grafts. J Oral Maxillofac Surg 1998;56:1073-1074.

[9] Buser D. 20 Years of guided bone regeneration in implant dentistry (Hardback), Quintessence Publishing Co Inc, US, 2009.

[10] Chiapasco M et al. Clinical outcome of autogenous bone blocks or GBR with e-PTFE membranes for the reconstruction of narrow edentulous ridges. Clin Oral Implants Res 1999;10:278-88.

[11] Chiapasco M, Romeo E, Casentini P, Rimondini L. Alveolar distraction osteogenesis vs. vertical guided bone regeneration for the correction of vertically deficient edentulous ridges: A 1-3-year prospective study on humans Clin Oral Implants Res 2004;15:82-95.
[12] Chiapasco M, Zaniboni M. Clinical outcomes of GBR procedures to correct peri implant dehiscences and fenestrations: a systematic review. Clin Oral Implants Res 2009;20 Suppl 4:113-23.

[13] Dahlin C, Linde A, Gottlow J, Nyman S. Healing of bone defects by guided tissue regeneration. Plast Reconstr Surg 1988;81:672-6.

[14] Dahlin C, Lekholm U, Becker W et al. Treatment of fenestration and dehiscence bone defects around oral implants using the guided tissue regeneration technique: a prospective multicenter study. Int J Oral Maxillofac Implants 1995; 10:312-8.

[15] Fontana F et al. Clinical and histologic evaluation of allogenic Bone matrix versus autogenous bone chips associated with titanium reinforced e-PTFE membrane for vertical ridge augmentation. A prospective pilot study. Int J Oral Maxillofac Implants 2008;23:1003-12.

[16] Friedmann A, Strietzel FP, Maretzki B, Pitaru S, Bernimoulin JP. Histological assessment of augmented jaw bone utilizing a new collagen barrier membrane compared to a standard barrier membrane to protect a granular bone substitute material: a randomized clinical trial. Clin Oral Implants Res 2002;13:587-94.

[17] $\mathrm{Fu} \mathrm{JH}$ et al. Horizontal bone augmentation: the decision tree. Int J Periodontics Restorative Dent 2011;31:429-36.

[18] Gentile P, Chiono V, Tonda-Turo C, Ferreira AM, Ciardelli G. Polymeric membranes for guided bone regeneration. Biotechnol J 2011; 6(10):1187-97.

[19] Geurs NC, Korostoff JM, Vassilopoulos PJ, Kang TH, Jeffcoat M, Kellar R, Reddy MS. Clinical and histologic assessment of lateral alveolar ridge augmentation using a synthetic long-term bioabsorbable membrane and an allograft. J Periodontol 2008;79(7):1133-40.

[20] Hämmerle CH, Jung RE. Bone augmentation by means of barrier membranes. Periodontol 2000 2003;33:36-53.

[21] Hämmerle CH, Lang NP. Single stage surgery combining transmucosal implant placement with guided bone regeneration and bioresorbable materials. Clin Oral Implants Res 2001;12(1):9-18.

[22] Hämmerle CH, Fourmousis I, Winkler JR, Weigel C, Brägger, U, Lang NP. Successful bone fill in late peri-implant defects using guided tissue regeneration. A short communication. J Periodontol 1995;66(4):303-308

[22] Hämmerle CH, Jung R, Yaman D, Lang N. Ridge augmentation by applying bioresorbable membranes and deproteinized bovine bone mineral: a report of twelve consecutive cases. Clin Oral Implants Res 2008;19(1):19-25.

[24] Hjorting-Hansen E. Bone grafting to the jaws with special reference to reconstructive preprosthetic surgery. A historical review. Mund Kiefer Geichtschir 2002,6:6-14.

[25] Huang, Qi, Liu, Xu. Construction of guided bone regeneration membrane by tissue engineering in vitro. Sheng $W u$ Yi Xue Gong Cheng Xue Za Zhi 2004, 21(4):579-81.

[26] Jovanovic SA, Schenk RK, Orsini M, Kenney EB. Supracrestal bone formation around dental implants: an experimental dog study. Int J Oral Maxillofac Implants 1995;10(1):23-31.

[27] Jensen S, Terheyden H. Bone augmentation procedures in localized defects in the alveolar ridge: clinical results with different bone grafts and bone-substitute materials. Int J Oral Maxillofac Implants 2009;24(suppl):218-236.

[28] Karring T, Nyman S, Gottlow J, Laurell L. Development of the biological concept of guided tissue regeneration-animal and human studies. Periodontol 2000 1993;1:26-35.

[29] Karring T, Nyman S, Lindhe J. Healing following implantation of periodontitis affected roots into bone tissue. J Clin Periodontol 1980;7(2):96-105.

[30] Kostopoulos L, Karring T. Guided bone regeneration in mandibular defects in rats using a bioresorbable polymer. Clin Oral Implants Res 1994;5:66-74.

[31] Lorenzoni M, Pertl C, Polansky RA, Jakse N, Wegscheider WA. Evaluation of implants placed with barrier membranes. Clini Oral Implants Res 2002;13:3 274-80.

[32] Machtei EE. The effect of membrane exposure on the outcome of regenerative procedures in humans: a meta-analysis. $J$ Periodontol 2001;72,4;512-6.

[33] Melcher AH. On the repair potential of periodontal tissues. J. Periodontology 1976; 47(5):256-60.

[34] Mellonig JT et al. Guided bone regeneration of bone defects associated witti implants: an evidence-based outcome assessment. 
Int J Periodontics Restorative Dent 1995;15(2):168-85.

[35] Nowzari H, Slots J. Microbiologic and clinical study of polytetrafluoroethylene membranes for guided bone regeneration around implants. Int J Oral Maxillofac Implants 1995;10(1):67-73.

[36] Nyman S, Karring T, Lindhe J, Planten S. Healing following implantation of periodontitis affected roots into gingival connective tissue. J Clin Periodontology 1980;7(5):394-401.

[37] Nyman S, Lang NP, Buser D, Bragger U. Bone regeneration adjacent to titanium dental implants using guided tissue regeneration: a report of two cases. Int J Oral Maxillofac Implants 1990;5:9-14.

[38] Nyman S, Lindhe J, Karring T. Reattachment. New attachment. In: Lindhe J (ed). Textbook of clinical periodontology. Copenhague: Munksgaard, 1989:450-8.

[39] Pallesen L, Schou S, Aaboe M, Hjorting-Hansen E, Nattestad A, Melsen F. Influence of particle size on the early stages of bone regeneration; a histologic and stereologic study in rabbit calvarium. Int J Oral Maxillofac Implants 2002;17(4):498-506.

[40] Pietrokovski J, Massler M. Alveolar ridge resorption following tooth extraction. J Prosth Dent 1967;17,21-7.

[41] Pietrokovski J, Starinsky R, Arensburg B, Kaffe I. Morphologic characteristics of bony edentulous jaws. J Prosthodontics 2007;16,141-7.
[42] Polimeni G, Koo KT, Pringle GA, Agelan A, Safadi FF, Wikesjö UM. Histopathological observations of a polylactic acid-based device intended for guided bone/tissue regeneration. Clin Implant Dent Relat Res 2008;10(2):99-105.

[43] Rocchietta I, Fontana F, Simion M. Clinical outcomes of vertical bone augmentation to enable dental implant placement: a systematic review. J Clin Periodontol 2008;35(Suppl. 8):203-15.

[44] Sandberg E, Dahlin C, Linde A. Bone regeneration by the osteopromotion technique using bioabsorbable membranes: an experimental study in rats. Int J Oral Maxillofac Surg 1993;51:1106-14.

[45] Schroeder HE. Biopathologie des structures orales. Paris : CDP, 1986.

[46] Shropp L, Wenzel A, Kostopoulos L, Karring T. Bone healing and soft tissue contour changes following single-tooth extraction: a clinical and radiographic 12-month prospective study. Int J Periodontics Restorative Dent 2003;23:313-23.

[47] Schwarz F, Sahm N, Becker J. Impact of the outcome of guided bone regeneration in dehiscence-type defects on the long-term stability of peri-implant health: clinical observations at 4 years. Clin Oral Implants Res 2012;23(2):191-6.

[48] Simion M et al. GBR with an e-PTFE Membrane associated with DFDBA: Histologic and histochemical analysis in a human implant retrieved after 4 years of loading. Int J Periodontics Restorative Dent 1996;16(4):338-47.

[49] Springer NG, Terheyden H, Geiß S, Härle F, Hedderich J, Açil Y. Particulated bone grafts - effectiveness of bone cell supply. Clin Oral Implants Res 2004;15(2):205-12.

[50] Tal H, Kozlovsky A, Artzi Z, Nemcovsky CE, Moses O. Cross-linked and non-cross-linked collagen barrier membranes disintegrate following surgical exposure to the oral environment: a histological study in the cat. Clin Oral Implants Res 2008;19(8):760-6.

[51] Tonetti MS, Hämmerle C. Advances in bone augmentation to enable dental implant placement: Consensus Report of the Sixth European Workshop on Periodontology. J Clin Periodontology 2008;35:168-72.

[52] Umeda H, Kanemaru, Yamashita M, Ohno T, Suehiro A, Tamura Y, Hirano S, Nakamura T, Omori $\mathrm{K}$, Ito J. In situ tissue engineering of canine skull with guided bone regeneration. Acta Otolaryngol 2009;129(12):1509-18.

[53] Yeh $\mathrm{H}$ et al. GBR for fenestration defects in dental implants. Chang Gung Med J 2003;6:684-9.

[54] Zellin G, Gritli-Linde A, Linde A. Healing of mandibular defects with different biodegradable and non-biodegradable membranes: an experimental study in rats. Biomaterials 1995;16:601-9.

[55] Zitzmann NU, Schärer P, Marinello CP. Long-term results of implants treated with guided bone regeneration: a 5-year prospective study. Int J Oral Maxillofac Implants 2001;16(3):355-66.

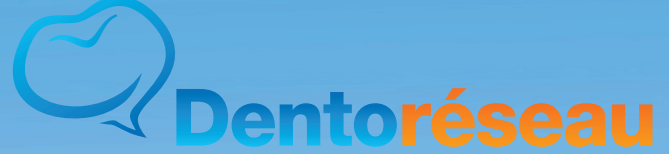

Le réseau social des chirurgiens-dentistes

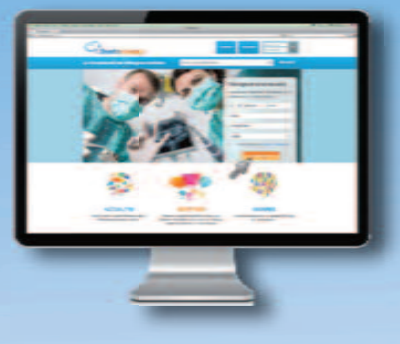

Découvrir et rejoindre une communauté de confrères

Participer et animer des groupes de discussion
Rejoignez le réseau social des chirurgiens-dentistes
Partager et actualiser ses connaissances

Créer et enrichir son réseau 